\title{
Development of a micro-satellite TSUBAME for X-ray polarimetry of GRBs
}

\author{
Yoichi Yatsu ${ }^{1}$, Mayumi Hayashi ${ }^{1}$, Kousuke Kawakami ${ }^{1}$, \\ Kazuki Tokoyoda ${ }^{1}$, Takahiro Enomoto ${ }^{1}$, Takahiro Toizumi ${ }^{1}$, \\ Nobuyuki Kawai ${ }^{1}$, Kazuya Ishizaka ${ }^{2}$, Azusa Muta ${ }^{2}$, \\ Hiroyuki Morishita ${ }^{2}$, Saburo Matsunaga ${ }^{2,3}$, Takeshi Nakamori ${ }^{4}$, \\ Jun Kataoka ${ }^{4}$, and Shin Kubo ${ }^{5}$ \\ ${ }^{1}$ Dept. of Physics, Tokyo Institute of Technology, \\ 2-12-1 Oookayama Meguro Tokyo, 152-8551, Japan \\ email: yatsu@hp.phys.titech.ac.jp \\ ${ }^{2}$ Dept. of Mechanical and Aerospace Engineering, Tokyo Institute of Technology, Japan \\ ${ }^{3}$ Dept. of Space Structure and Materials, Institute of Space and Astronautical Science, Japan \\ ${ }^{4}$ Research Institute for Science and Engineering, Waseda University, Japan \\ ${ }^{5}$ CLEAR PULSE CO., LTD., Japan
}

\begin{abstract}
Hard X-ray polarization is believed to be one of the most promising methods to investigate the physical processes just around the central engines by constraining the magnetic environment. For this purpose we are now developing a compact and highly sensitive hard X-ray polarimeter aboard a university class micro-satellite "TSUBAME". We are now developing the flight model of the satellite aiming for the launch in late 2012 from Russia.
\end{abstract}

Keywords. GRBs, Polarimetry, micro-satellite.

\section{Introduction}

The "fireball model" in which gamma-rays originate in a relativistic outflow driven by some energy source is commonly accepted for explaining gamma-ray bursts (GRBs). However the physical connection between the "fireballs" and the central engines is still unclear. It is often claimed that a magnetic field plays an important role in generating the relativistic outflow from the gravitational energy and, consequently, the gamma-ray emission. For this issue, X-ray/gamma-ray polarimetry is believed to be one of the most promising methods for providing information on the magnetic environment around the emitting region.

GRBs become much brighter than the Sun in the gamma-ray sky and they occur at a rate of about one per day in the universe. Therefore, if we can start observations soon after the detection, even a small detector mounted on a micro-satellite can provide enough information to constrain the physical mechanism in GRBs, and this fact was already clarified by the GAP aboard IKAROS (Yonetoku et al. 2011).

\section{Satellite and Detector System}

For this science mission we chose a $50 \mathrm{~kg}$ micro-satellite bus as a platform (Fig. 1). Although the mission payload is strictly limited in size, mass, and power supply the micro-satellite has a quite attractive advantage: launch opportunity. In order to perform "on-axis" pointing observations the satellite bus possesses a high speed attitude control system using compact and high-torque actuators, so-called "control moment gyroscopes". 


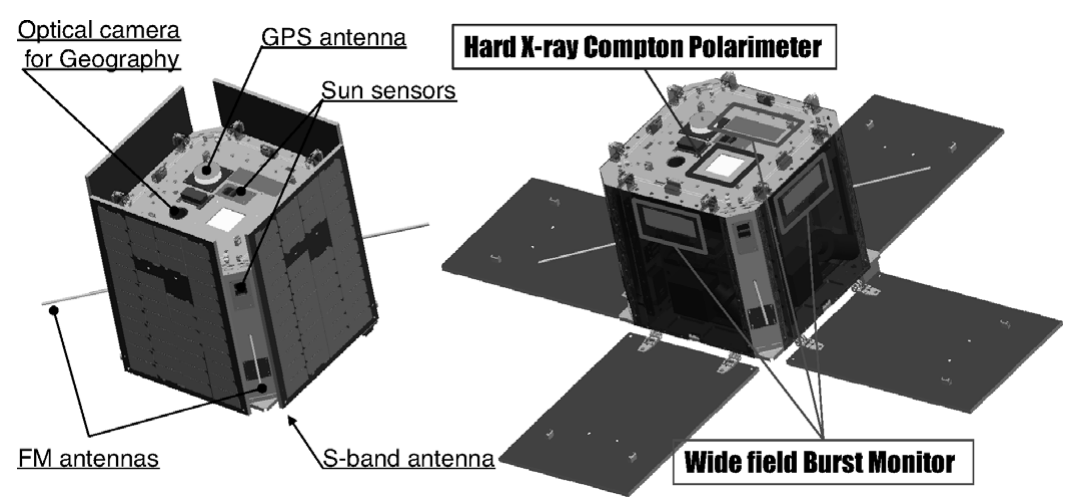

Figure 1. Overview of the micro-satellite TSUBAME (3D-CAD model).

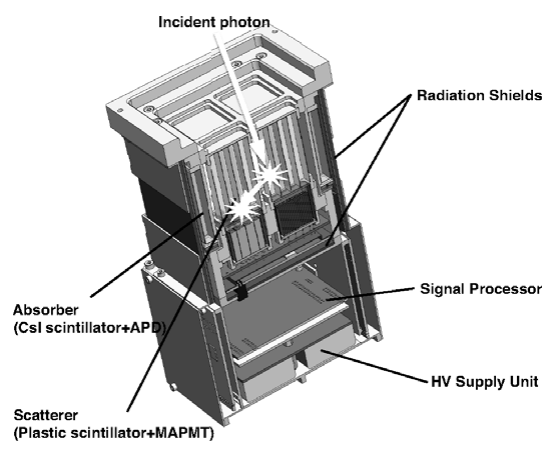

Figure 2. Schematic view of the hard X-ray Compton polarimeter.
Table 1. Expected performance of HXCP.

\begin{tabular}{lr}
\hline Energy band & \multicolumn{1}{c}{$30-200 \mathrm{keV}$} \\
\hline Field of view & $15^{\circ} \times 15^{\circ}$ \\
\hline Effective area & $7.1 \mathrm{~cm}^{2}$ at $100 \mathrm{keV}$ \\
\hline Modulation factor & $54 \%$ at $100 \mathrm{keV}$ \\
\hline $\mathrm{MDP}^{\mathrm{a}}$ & $6.2 \%$ for GRB021206 \\
\hline $\begin{array}{l}\text { a MDP is the minimum detectable polar- } \\
\text { ization with } 3 \sigma \text { confidence level. Detailed } \\
\text { information can be found in Yatsu et al. } \\
(2011) .\end{array}$
\end{tabular}

These that enable high speed attitude control faster than $6^{\circ} \mathrm{s}^{-1}$. Thanks to a wide field burst monitor for real time position determination of GRBs, TSUBAME can start a pointing observation within $\sim 15$ s after the detection for any GRB in the half-sky field of view of the burst monitor.

The main detector, the Hard X-ray Polarimeter (Fig. 2) measures X-ray polarization utilizing anisotropy of the Compton scattering. The expected performance of the HXCP is summarized in Table. 1. While the WBM always monitors half the sky and if a GRB detected WBM calculate the coordinate with on-board MPU.

\section{References}

Yatsu, Y., Enomoto, T., Kawakami, K., Tokoyoda, K., Toizumi, T., Kawaia, N., Ishizaka, K., Matsunaga,S., Nakamori, T., Kataoka, J., \& Kubo, S. 2011, SPIE, Volume 8145, pp. 814508814508-11

Yonetoku, D., Murakami, T., Gunji, S., Mihara, T., Toma, K., Sakashita, T., Morihara, Y., Takahashi, T., Toukairin, N., Fujimoto, H., Kodama, Y., Kubo, S. et al. 2011, ApJ, 743, Issue2, L30 